\title{
DETERMINAÇÃO DIRETA DE MOLIBDÊNIO EM COMPRIMIDOS POLIVITAMÍNICOS POR VOLTAMETRIA EM MEIO NÃO AQUOSO
}

\author{
Alex M. de Almeida \\ Instituto de Química, Universidade Federal de Uberlândia, 38400-902 Uberlândia - MG \\ Luiz M. Aleixo ${ }^{\dagger}$, João Carlos de Andrade* e Aline Renée Coscione \\ Instituto de Química, Universidade Estadual de Campinas, CP 6154, 13084-971 Campinas - SP
}

Recebido em 27/10/04; aceito em 4/4/05; publicado na web em 10/8/05

\begin{abstract}
DIRECT DETERMINATION OF MOLYBDENUM IN POLYVITAMINIC TABLETS BY VOLTAMMETRY IN NON-AQUEOUS MEDIA. Adsorptive stripping voltammetry carried out in a homogeneous ternary solvent composed of N,N-dimethylformamide, water and ethanol, with $\alpha$-benzoinoxime $(\alpha \mathrm{BO})$ as the complexing agent for $\mathrm{Mo}(\mathrm{VI})$ and a $0.5 \mathrm{~mol} \mathrm{~L}^{-1}$ acetic acid - sodium acetate buffer as supporting electrolyte was successfully used for the determination of molybdenum in polyvitamin-polymineral tablets. Tablet samples were analyzed and the results were compared with those obtained both by graphite furnace atomic absorption and by recovery tests, with good correlations, indicating that this may be considered as an alternative procedure for routine determination of $\mathrm{Mo}(\mathrm{VI})$ in pharmaceutical samples.
\end{abstract}

Keywords: molybdenum determination; pharmaceuticals; voltammetry.

\section{INTRODUÇÃO}

O molibdênio é um elemento essencial para a nutrição de animais e plantas, estando envolvido em vários processos catalíticos e conversões metabólicas ${ }^{1-5}$. Complexos contendo molibdênio são também administrados no tratamento de algumas doenças, como a do mal de Wilson ${ }^{6-9}$, que é caracterizada por um aumento de cobre no organismo devido à ausência da enzima ATPase que atua na eliminação de cobre. $\mathrm{O}$ complexo tetratiomolibdato é geralmente administrado ao paciente para substituir a enzima no processo de eliminação do cobre. Esse mesmo composto parece suprimir tumores ${ }^{10}$. Por isso, o molibdênio vem sendo incluído na composição de diversos polivitamínicos poliminerais, com objetivo de suprir possíveis deficiências desse elemento no organismo humano.

Os métodos analíticos empregados para determinação de Mo(VI) estão geralmente sujeitos a interferências químicas, de modo que procedimentos de extração e de pré-concentração são freqüientemente empregados nessas determinações. A inclusão dessas etapas torna os procedimentos analíticos lentos e requer atenção especial durante as operaçãoes realizadas no laboratório. A determinação de molibdênio em medicamentos pode ser ainda mais complicada, devido às quantidades relativamente altas de outros componentes presentes nas formulações.

A espectrometria atômica é empregada preferencialmente na quantificação de $\mathrm{Mo}(\mathrm{VI})$ mas, devido às características refratárias desse elemento, apenas os métodos de absorção atômica em forno de grafite (GFAAS) e plasma de argônio acoplado indutivamente (ICP-OES) têm utilidade prática. Como conseqüência, são altamente desejáveis alternativas que permitam a determinação direta de Mo(VI) através de técnicas analíticas mais baratas, principalmente para uso em análises de rotina, como no controle de qualidade de medicamentos.

*e-mail: dandrade@iqm.unicamp.br

$\dagger$ In memoriam
Recentemente, a voltametria adsortiva (AdSV) foi empregada com sucesso na determinação de molibdênio em amostras complexas usando um sistema ternário homogêneo de solventes (STHS), composto por N,N-dimetilformamida (DMF), água e etanol ${ }^{11,12}$. Esse método mostrou-se altamente sensível ao $\mathrm{Mo}(\mathrm{VI})$ e pouco sujeito a interferências químicas, uma vez que a ação das espécies interferentes é minimizada (ou até eliminada) nas etapas de diluição necessárias para se atingir a faixa de calibração empregada na determinação. Além disso, procedimentos de modelagem multivariada ${ }^{12}$ mostraram que as fortes interações causadas por fósforo (na forma de fosfato) e ferro (na forma $\mathrm{Fe}^{3+}$ ) são grandemente compensadas quando ambas as espécies se encontram em solução, tormando o sistema AdSV-STHS muito menos susceptível ao efeito de ferro e fosfato do que seria esperado. Tungstênio (na forma de W(VI)) e vanádio (na forma de $\mathrm{V}(\mathrm{V})$ ) são também interferentes potenciais do método, mas essas espécies estão geralmente ausentes em medicamentos. Isso nos levou a aplicar esse procedimento analítico para determinação de Mo(VI) em amostras de comprimidos polivitamínicos poliminerais. O procedimento proposto apresenta vantagens em relação à técnica com forno de grafite (GFAAS) e pode ser aplicado na determinação de $\mathrm{Mo}(\mathrm{VI})$ em outros medicamentos que contenham molibdênio, sendo adequado para determinações em condições de rotina.

\section{PARTE EXPERIMENTAL}

Todos os reagentes utilizados foram de grau analítico e água deionizada foi empregada na preparação de todas as soluções. As soluções foram armazenadas em frascos de polietileno de alta densidade e os volumes foram dispensados utilizando-se buretas ou micropipetas calibradas.

A solução estoque de $1.000 \mathrm{mg} \mathrm{L}^{-1}$ de molibdênio foi preparada através da diluição do conteúdo de uma ampola de padrão MerckTritisol para $1.000 \mathrm{~mL}$ com água. Uma solução de 4,0 x $10^{-4} \mathrm{~mol} \mathrm{~L}^{-1}$ de $\alpha$-benzoinoxima ( $\alpha \mathrm{BO})$ foi preparada por dissolução do reagente na quantidade apropriada de N,N'-dimetilformamida (DMF). 
O STHS empregado como meio para determinação de Mo(VI) por AdSV foi uma mistura composta por $37,5 \% \mathrm{~m} / \mathrm{m} \mathrm{DMF}, 35,3 \%$ $\mathrm{m} / \mathrm{m}$ etanol e $27,2 \% \mathrm{~m} / \mathrm{m}$ água, essa última contendo o eletrólito de suporte (solução tampão de acetato de sódio-ácido acético) e uma alíquota apropriada da solução de trabalho de molibdênio ou de $\operatorname{amostra}^{11,12}$. Para facilitar a preparação das soluções, as massas desses solventes foram transformadas em volumes, considerandose sua concentração relativa na solução homogênea e a densidade da mistura de solventes e de seus componentes individuais. Essa aproximação não afeta significativamente os resultados finais, pois a composição do solvente é mantida constante. Os volumes correspondentes dos solventes, empregados no preparo da solução STHS, são indicados no procedimento abaixo.

\section{Preparação da amostra}

Três marcas diferentes de comprimidos polivitamínicos poliminerais foram identificadas neste trabalho como amostras 1 , 2 e 3. Três comprimidos de cada marca foram analisados. Cada comprimido foi pesado com precisão de $\pm 0,1 \mathrm{mg}$ e dissolvido em $20,0 \mathrm{~mL}$ de uma solução $1,00 \mathrm{~mol} \mathrm{~L}^{-1} \mathrm{de} \mathrm{HCl}$, sob aquecimento brando em um béquer de $50 \mathrm{~mL}$, até sua completa dissolução. A solução resultante foi transferida quantitativamente para um balão volumétrico de $50 \mathrm{~mL}$ após filtragem. O papel de filtro foi lavado com uma pequena porção do mesmo ácido completando-se, em seguida, o volume com esse mesmo ácido.

\section{Medidas e determinações}

As medidas de voltametria adsortiva (AdSV) foram feitas empregando-se varreduras de potencial entre -1050 e $-1400 \mathrm{mV}$, no modo voltametria de pulso diferencial, com amplitude de pulso de $50 \mathrm{mV}$, tempo de acumulação de $150 \mathrm{~s}$ e duração de pulso de 40 ms. O equipamento utilizado foi um polarógrafo Radiometer Copenhagen, modelo Pol 150, acoplado a um stand polarográfico Radiometer Copenhagen 150, equipado com uma cela contendo três eletrodos: um eletrodo de gota pendente de mercúrio (HMDE) como eletrodo de trabalho, um eletrodo de referência de $\mathrm{Ag} / \mathrm{AgCl}$ e um fio de platina como eletrodo auxiliar.

Os experimentos foram realizados usando-se uma cela voltamétrica de $10 \mathrm{~mL}$, sob atmosfera de $\mathrm{N}_{2}$, obtida após um tempo de purga de 5 min. A aquisição de dados e o registro das varreduras foram feitas através do uso do software Trace Master (version 2.03), fornecido pela Radiometer.

Quatro mililitros $( \pm 0,01 \mathrm{~mL})$ de cada amostra dissolvida ou da solução de calibração foram transferidos para fracos "snap-cap" de $80 \mathrm{~mL}$ e misturados com $2,00 \mathrm{~mL}$ de uma solução $1,00 \mathrm{~mol} \mathrm{~L}^{-1}$ de hidróxido de sódio, seguido pela adição de $1,7 \mathrm{~mL}$ de uma solução $0,5 \mathrm{~mol} \mathrm{~L}^{-1}$ de tampão ácido acético-acetato de sódio (eletrólito de suporte), 0,8 $\mathrm{mL}$ de água, 13,0 $\mathrm{mL}$ de etanol, 10,0 $\mathrm{mL}$ de DMF e 2,0 mL da solução de $\alpha \mathrm{BO}$ preparada em DMF. Depois de homogeneizar, $10,00 \mathrm{~mL}$ da solução contendo a amostra (ou o padrão de calibração) foram transferidos para a cela e a varredura voltamétrica foi realizada. O pico de potencial devido ao complexo Mo- $\alpha$ BO ocorre aproximadamente em $-1250 \mathrm{mV}$, onde os picos de corrente foram medidos.

Testes de recuperação também foram realizados para comparação, além das determinações diretas de Mo por AdSV. Isso foi feito através da adição de pequenos volumes, bem definidos, de solução padrão estoque de $\mathrm{Mo}(\mathrm{VI})$ a $25,00 \mathrm{~mL}$ da amostra dissolvida em $\mathrm{HCl}$, na proporção de $100 \mu \mathrm{g}$ Mo(VI) por g de comprimido. Alíquotas da solução resultante foram tomadas para preparar as soluções do STHS para as medidas, como descrito acima.
O Mo(VI) contido nas amostras também foi determinado por absorção atômica em forno de grafite (GFAAS), para comparação dos resultados obtidos por voltametria. O equipamento empregado foi um Perkin Elmer GFAAS, modelo AA Winlab, com correção de fundo baeada no efeito Zeeman. As determinações foram feitas na linha de Mo a $313,3 \mathrm{~nm}$, usando-se uma solução de paládio - magnésio como modificador químico ${ }^{13}$. A exatidão do método foi verificada através da comparação dos resultados obtidos por voltametria com aqueles obtidos por absorção atômica e testes de recuperação.

\section{RESULTADOS E DISCUSSÃO}

O modelamento do comportamento de fósforo e ferro na determinação de Mo(VI), empregando as condições experimentais utilizadas no sistema AdSV-STHS ${ }^{12}$, mostrou que a interação entre essas espécies no sistema químico constituído pela mistura de solventes é significativa e esse efeito reduz as interferências das espécies individuais em uma faixa de concentrações bem maior que verificado inicialmente. Isso permite propor o procedimento simples e direto descrito neste trabalho como uma alternativa viável para determinação direta de molibdênio em comprimidos polivitamínicos poliminerais.

Apesar da composição usualmente complexa dos medicamentos testados, não há picos de corrente de espécies que possam sobrepor os picos do complexo Mo- $\alpha \mathrm{BO}$, causando interferência na região de potencial empregada para determinação de $\mathrm{Mo}(\mathrm{VI})$. Porém, uma vez que a elevada corrente de fundo observada nessas amostras pode perturbar a determinação precisa dos picos de corrente para o molibdênio, realizaram-se também testes de recuperação. Os perfis voltamétricos típicos obtidos para as amostras analisadas, antes e depois da adição do padrão de Mo(VI), são mostrados na Figura 1 e indicam que essa possível sobreposição não é um problema.

Uma curva de calibração característica (corrente de pico vs. concentração de $\mathrm{Mo}(\mathrm{VI})$ ) segue a equação polinomial dada por: I $=0,223+0,216\left[\mathrm{C}_{\mathrm{Mo}(\mathrm{VI})}\right]-0,011\left[\mathrm{C}_{\mathrm{Mo}(\mathrm{VI})}\right]^{2}\left(\mathrm{r}^{2}=0,997\right)$, onde I é o valor absoluto de corrente em $\mu \mathrm{A}$ e $\mathrm{C}_{\mathrm{Mo}(\mathrm{VI})}$ é a concentração de Mo em $\mu \mathrm{g} \mathrm{L}^{-1}$. As variações na composição do STHS e os ajustes nas condições operacionais introduzidas neste trabalho não alteram significativamente as figuras de mérito determinadas anteriormente ${ }^{11}$.

Os resultados obtidos para os três medicamentos testados são mostrados na Tabela 1, juntamente com os resultados obtidos por GFAAS e os valores declarados pelo fabricante. Podese verificar que os resultados obtidos por AdSV apresentam boa concordância com aqueles obtidos por GFAAS, confirmando a boa precisão e exatidão obtidas com o sistema AdSV-STHS. Esses dados mostram também que a quantidade de Mo(VI) pre-

Tabela 1. Resultados das determinações de Mo(VI) nas amostras, obtidos por voltametria em meio não aquoso e por GFAAS

\begin{tabular}{crrr}
\hline Amostras & \multicolumn{2}{c}{ Mo(VI) $/ \mu \mathrm{g} \mathrm{g}^{-1}$} & $\begin{array}{c}\text { Valor declarado } \\
\text { pelo fabricante } \\
\left(\mu \mathrm{g} \mathrm{g}^{-1}\right)^{* *}\end{array}$ \\
\hline 1 & Voltametria * $^{*}$ & \multicolumn{1}{c}{ GFAAS * $^{*}$} & \multicolumn{1}{c}{$225,5 \pm 3,8$} \\
2 & $227,3 \pm 4,0$ & $221,7 \pm 6,9$ & $79,1 \pm 1,9$ \\
3 & $78,5 \pm 2,0$ & $80,4 \pm 3,8$ & $40,6 \pm 0,2$ \\
\hline
\end{tabular}

(*) Valores médios, calculados para amostras em triplicata; ${ }^{(*)}$ média da quantidade de Mo nas amostras testadas, calculadas com base na massa do comprimido e no valor declarado pelo fabricante 
Tabela 2. Resultados do teste de recuperação usando o método voltamétrico proposto

\begin{tabular}{ccccr}
\hline Amostras & Mo(VI) encontrado / $\mu \mathrm{g} \mathrm{g}^{-1}$ & $\mathrm{Mo}(\mathrm{VI})$ adicionado / $\mu \mathrm{g} \mathrm{g} \mathrm{g}^{-1}$ & Mo(VI) total & ${\text { Recuperação }(\%) / \mu \mathrm{g} \mathrm{g}^{-1}}$ \\
\hline 1 & $227,3 \pm 4,0$ & 100,0 & $326,9 \pm 4,5$ & 99,7 \\
2 & $78,5 \pm 2,0$ & 100,0 & $178,3 \pm 1,1$ & 99,8 \\
3 & $48,9 \pm 1,6$ & 100,0 & $149,1 \pm 2,2$ & 100,2 \\
\hline
\end{tabular}
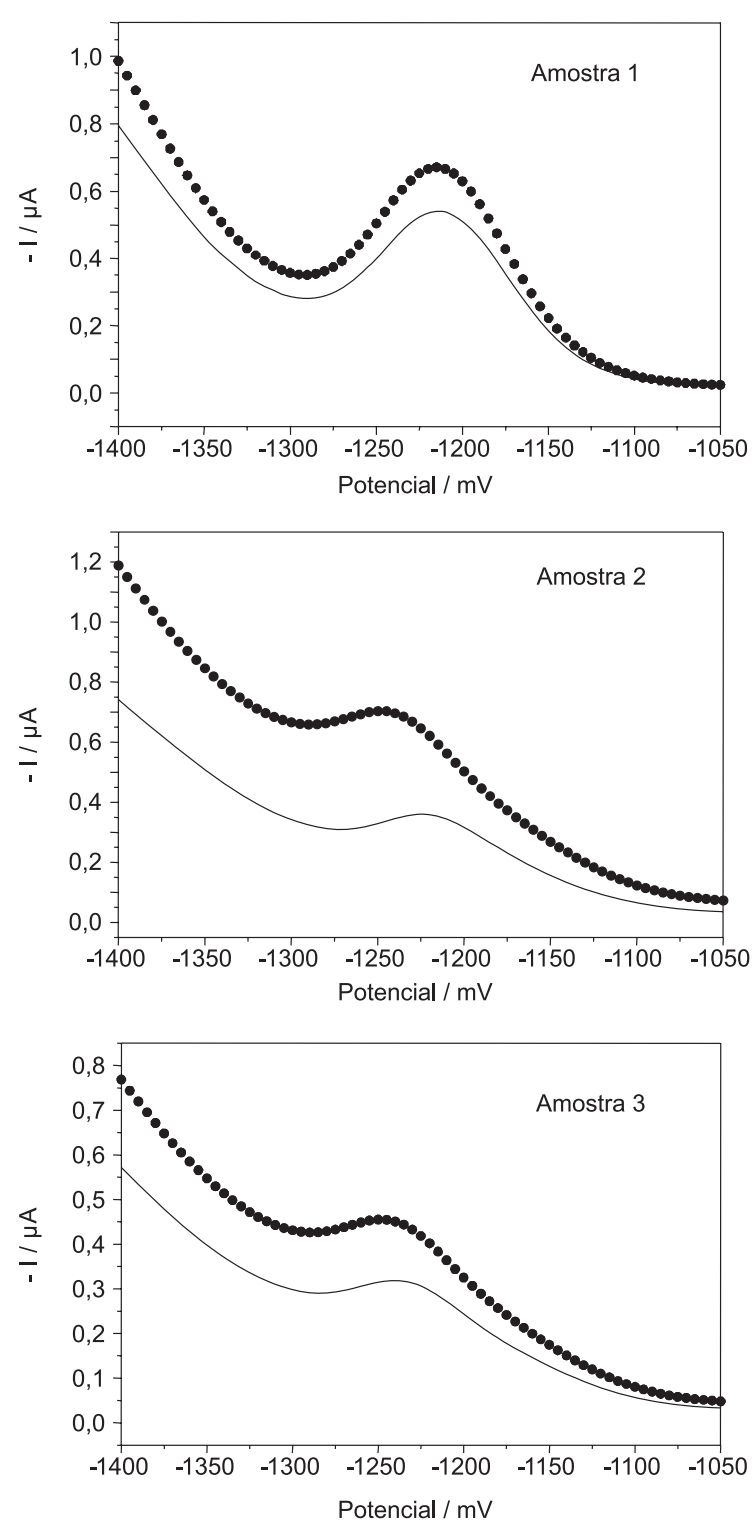

Figura 1. Voltamograma das amostras dos comprimidos polivitamínicos polimimerais 1, 2 e 3, mostrando os picos do complexo Mo - $\alpha B O$ observados nas

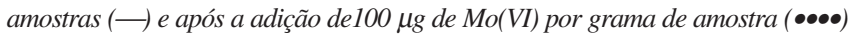

sente na amostra 3 é cerca de $17 \%$ maior que o reportado pelo fabricante. A Tabela 2 mostra as concentrações e a porcentagem de recuperação obtidas nos testes de recuperação para essas amostras. Os valores mostram que a determinação de molibdênio pode ser feita diretamente, sem a necessidade da adição de um padrão interno.

A partir desses resultados, mostrou-se que o método de determinação de $\mathrm{Mo}(\mathrm{VI})$ por voltametria adsortiva proposto pode ser considerado como uma alternativa simples e direta para determinação dessa espécie química em amostras de medicamentos. Além disso, devido à sua elevada detectabilidade e boa exatidão, as amostras podem ser analisadas sem a utilização de etapas de separação ou pré-concentração, empregando-se uma técnica bem mais barata que a espectrometria com forno de grafite e mais facilmente adaptável às condições das determinações de rotina.

\section{AGRADECIMENTOS}

Ao CNPq pela bolsa concedida a A. M. Almeida. Os autores agradecem também ao Dr. E. R. Pereira Filho e ao Prof. M. A. Z. Arruda pelo uso do GFAAS, e à Profa. C. H. Collins pelas sugestões.

\section{REFERÊNCIAS}

1. Burgmayer, S. J. N.; Stiefel, E. I.; J. Chem. Educ. 1985, 62, 943.

2. Sagi, M.; Omarov, R. T.; Lips, S. H.; Plant Sci. 1998, 135, 125.

3. Sagi, M.; Lips, H. S.; Plant Sci. 1998, 135, 17.

4. Barabás, N. K.; Oramov, R. T.; Erdei, L.; Lips, S. H.; Plant Sci. 2000, 155, 49.

5. Sauer; P.; Frébortová, J.; Sebela, M.; Galuska, P.; Jacobsen, S.; Pec, P.; Frébort, I.; Plant Physiol. Biochem. 2002, 40, 393.

6. Schilsky, M. L.; Seminars Liver Disease 1996, 16, 83.

7. Brewer, G. J.; Dick, R. D.; Yuzbasiyan-Gurkin, V.; Tankanow, R.; Young, A. B.; Kluin, K. J.; Arch. Neurol. 1991, 48, 42.

8. Brewer, G. J.; Johnson, V.; Dick, R. D.; Kluin, K. J.; Fink, J. K.; Brunberg,J. A.; Arch. Neurol. 1996, 53, 1017.

9. Karunajeewa, H.; Wall, A.; Metz, J.; Grigg, A.; Aus. NZ J. Med. 1998, 28, 215.

10. Pan, Q.; Kleer, C. G.; van Golen, K. L.; Irani, J.; Bottema, K. M.; Bias, C.; de Carvalho, M.; Mesri, E. A.; Robins, D. M.; Dick, R. D.; Brewer, G. J.; Merajver, S. D.; Cancer Res. 2002, 62, 4854.

11. de Andrade, J. C.; Almeida, A. M.; Coscione, A. R.; Aleixo, L. M.; Analyst 2001, 126, 892.

12. de Andrade, J. C.; Almeida, A. M.; Aleixo, L. M.; Coscione, A. R.; de Abreu, M. F.; Anal Chim. Acta 2003, 487, 243.

13. The THGA Graphite Furnace: Techniques and Recommended Conditions, Release 1.2, Perkin Elmer: Germany, 1995, p. 7-35. 